\title{
Experiments to Test the A-UGV Capabilities Standard
}

\author{
Soocheol Yoon \\ Roger Bostelman \\ Ann Virts
}

This publication is available free of charge from: https://doi.org/10.6028/NIST.IR.8407 


\title{
Experiments to Test the A-UGV Capabilities Standard
}

\author{
Soocheol Yoon, Ph.D. \\ Guest Researcher, Mobility Performance of Robotic Systems Project \\ Intelligent Systems Division \\ Engineering Laboratory \\ Roger Bostelman, Ph.D. \\ Research Associate, Mobility Performance of Robotic Systems Project \\ Intelligent Systems Division \\ Engineering Laboratory \\ Visiting Researcher \\ University of Maryland Institute for Advanced Computer Studies \\ College Park, MD 20742 \\ Ann Virts \\ Project Leader, Mobility Performance of Robotic Systems Project \\ Intelligent Systems Division \\ Engineering Laboratory
}

This publication is available free of charge from: https://doi.org/10.6028/NIST.IR.8407

December 2021

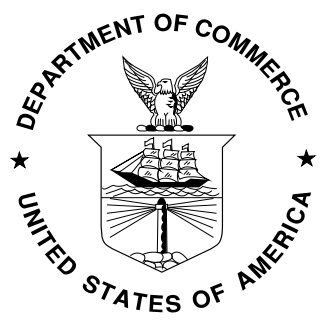

U.S. Department of Commerce Gina M. Raimondo, Secretary

National Institute of Standards and Technology James K. Olthoff, Performing the Non-Exclusive Functions and Duties of the Under Secretary of Commerce for Standards and Technology \& Director, National Institute of Standards and Technology 
Certain commercial entities, equipment, or materials may be identified in this document in order to describe an experimental procedure or concept adequately. Such identification is not intended to imply recommendation or endorsement by the National Institute of Standards and Technology, nor is it intended to imply that the entities, materials, or equipment are necessarily the best available for the purpose.

National Institute of Standards and Technology Interagency or Internal Report 8407 Natl. Inst. Stand. Technol. Interag. Intern. Rep. 8407, 17 pages (December 2021)

This publication is available free of charge from: https://doi.org/10.6028/NIST.IR.8407 


\begin{abstract}
Automatic, Automated, or Autonomous - Unmanned Ground Vehicles (A-UGVs), as referred to by ASTM International Committee F45, are industrial vehicles that have capabilities to navigate, dock, avoid obstacles, and interact with other vehicles and systems all within intended environments (e.g., warehouses, hospitals, outdoor facility perimeters) set by the manufacturer. A new ASTM F45 standard is near the ballot stage to serve as a guide for providing a basis for A-UGV manufacturers and users to compare capabilities between AUGVs. The guide includes a report for documenting that the user has proven or asserted a capability, not tested the capability, or that the A-UGV does not have the capability. Although an infinite number of A-UGV tests are possible, this paper provides three cases that test the new standard's effectiveness to correctly define A-UGV capabilities. In one case experiments showed that several capabilities as written in the standard were correctly demonstrated. Alternatively, two cases demonstrated through experiments that the A-UGV capabilities as written in the standard may need improvements and as such, the paper provides recommendations for modifying the standard.
\end{abstract}

\title{
Key words
}

ASTM F45; A-UGV; A-UGV capabilities; autonomous industrial vehicles; standards; performance measurement. 


\section{Table of Contents}

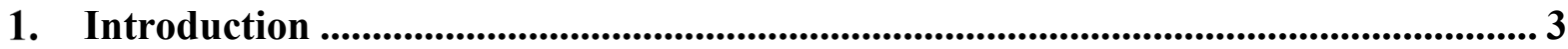

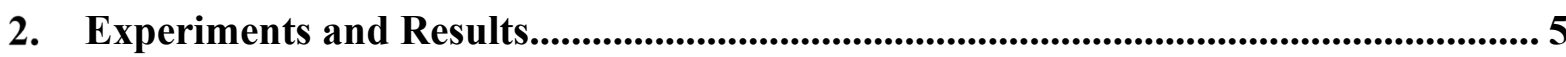

2.1. Experiment 1: A-UGVs within the same zone ..................................................... 5

2.2. Experiment 2: Transparent obstacles in front of another obstacle ............................. 7

2.3. Experiment 3: Loaded A-UGV on a ramp ………........................................... 9

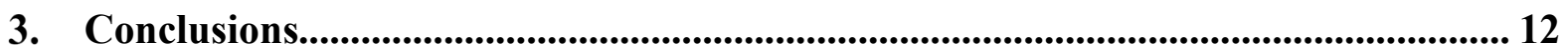

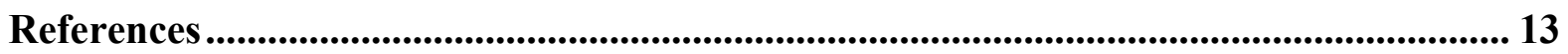

\section{List of Tables}

Table 1. Experiment 1 test results showing the A-UGV success rate and test occurrences. .... 6 Table 2. Number of times that the A-UGV reached the goal comparing A-UGV set speed vs. payload on the $5^{\circ}$ ramp. 11 Table 3. Number of times that the A-UGV reached the goal comparing A-UGV set speed vs. payload on the $10^{\circ}$ ramp. 11

\section{List of Figures}

Fig. 1. Snapshot of the A-UGV Capabilities standard test report where all boxes must be addressed, but one capability does not rely on other capabilities.

Fig. 2. Layout (left) and actual path (right) confined by blocks for two A-UGVs in a narrow path test.

Fig. 3. When two A-UGVs choose different sides (left) and the same side (right)................. 7 Fig. 4. Transparent curtain (top-left - marked by the red dashed line) is detected (top-right) as an obstacle causing the A-UGV to stop. Obstacle is placed behind a transparent curtain (bottom-left) and both are detected, respectively (Bottom-right)............................................ 8

Fig. 5. Summary of experimental setup for a loaded A-UGV while navigating on ramps. ... 10 


\section{Introduction}

There is a growing interest in autonomous industrial vehicles and investigations into ways to introduce them into the real world are also being conducted in various other domains. For example, Starship and George Mason University built an outdoor mobile robot to deliver food and drinks [1]. Agricultural [2] and medical/healthcare [3] applications are also actively adopting mobile robots. There are also many cases in the public service domain using mobile robots to guide people, such as airport passenger assistance [4], and for cleaning commercial spaces [5]. Beyond systems of the past with preprogrammed guide paths, i.e., automatic guided vehicles (AGVs) [6], manufacturing has also moved forward with vehicles that are now referred to as autonomous mobile robots (AMRs) [7]. These newer vehicles operate in minimally structured or unstructured environments requiring varying degrees of autonomy. Regardless of the application domain, autonomous vehicles must abide by safety standards [8]. Complementing and supporting safety standards are performance standards that ensure that these vehicles demonstrate the capabilities to perform the tasks that are specified by the manufacturer.

ASTM International Committee F45 [9] has been developing performance standards for autonomous industrial vehicles since 2014. Several performance standards have been published and many are currently considered work items under development. Published F45 standards address: navigation, obstacle description and detection, environmental conditions, vehicle configuration, and terminology. Current F45 work items are addressing: docking, position measurement, communication impairments, fleets, combining standards, and capabilities. Unlike AGVs and AMRs, which have varying degrees of autonomy, F45 combines these vehicle type names into a single term: Automatic, Automated, or AutonomousUnmanned Ground Vehicles (A-UGVs). This is because example vehicle capabilities such as following a preprogrammed guide path (which is typically associated with AGVs) can also be used by AMRs to reach a goal in certain cases. Therefore, no distinction is made for performance tests of the many varied A-UGVs until their varying autonomous capabilities require different tests. The question then becomes, how can the A-UGV capabilities be defined such that manufacturers and users can fully demonstrate the capabilities of their vehicles, no matter what the vehicles are called?

In 2018, F45 began developing a standard guide, called A-UGV Capabilities, to decompose the basic functionality of automatic through autonomous vehicle capabilities based on a set of categories, such as navigation, docking, obstacle avoidance, fleets, etc. Since its inception, the capabilities standard has progressed beyond additive levels of autonomy (i.e., levels that build on top of lower levels) into simply labeling them as varying capabilities [10]. The standard guide not only defines capabilities but will also serve as a roadmap of F45 standard performance test methods to be developed. The guide includes a report for documenting that the user has proven (i.e., from a standard test method) or asserted (i.e., not from a standard test method) a capability, has not tested the capability, or that the A-UGV does not have the capability. Figure 1 shows a snapshot of the test report where all boxes must be addressed, and that one capability does not rely on other capabilities. 


\begin{tabular}{|c|c|c|c|c|c|c|c|}
\hline \multicolumn{2}{|c|}{ CATEGORY } & \multicolumn{6}{|c|}{ A-UGV CAPABILITIES } \\
\hline \multirow{2}{*}{\multicolumn{2}{|c|}{$\begin{array}{l}\text { Goal Navigation: } \\
\text { In situ }\end{array}$}} & \multicolumn{2}{|c|}{$\begin{array}{l}\text { Can determine and } \\
\text { navigate an initial path to } \\
\text { the goal }\end{array}$} & \multicolumn{2}{|c|}{$\begin{array}{l}\text { If blocked, can determine } \\
\text { and navigate an alternate } \\
\text { path to the goal. E.g., } \\
\text { navigate a different } \\
\text { hallway/aisle }\end{array}$} & \multicolumn{2}{|c|}{$\begin{array}{l}\text { If blocked, can determine } \\
\text { and navigate multiple } \\
\text { alternate paths to the } \\
\text { goal. E.g., navigate a } \\
\text { third hallway/aisle if the } \\
\text { second is blocked }\end{array}$} \\
\hline & & & Config: __ & & Config: __ & & Config: __ \\
\hline \multirow{3}{*}{ Yes } & Proven & Date: & Std: & \multicolumn{2}{|c|}{ Date: _ Std: } & \multicolumn{2}{|c|}{ Date: _ Std: } \\
\hline & Asserted & \multicolumn{2}{|l|}{ Date: } & \multicolumn{2}{|l|}{ Date: } & \multicolumn{2}{|l|}{ Date: } \\
\hline & Untested & \multicolumn{2}{|l|}{$\sqrt{1}$} & \multicolumn{2}{|l|}{$\sqrt{1}$} & \multicolumn{2}{|l|}{ 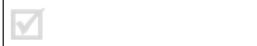 } \\
\hline & No & \multicolumn{2}{|l|}{$\sqrt{7}$} & \multicolumn{2}{|l|}{$\sqrt{1}$} & \multicolumn{2}{|l|}{$\sqrt{1}$} \\
\hline
\end{tabular}

Fig. 1. Snapshot of the A-UGV Capabilities standard test report where all boxes must be addressed, but one capability does not rely on other capabilities.

Examples of the report are described here. Figure 1 shows the "Goal Navigation: In situ" category, which refers to the A-UGV's ability to navigate to a goal while planning a path in real-time (i.e., not pre-programmed). For this category, the independent capabilities are that the A-UGV can determine and navigate an initial path to the goal or find one or more alternative paths if the original planned path to the goal is blocked. Within the "Obstacle Avoidance" category, capabilities are that the A-UGV can stop if the path is obstructed or can navigate around static or moving obstacles without collision. Within the "Environmental Conditions" category, if using ASTM F3218 [11], environmental conditions under which the A-UGV can operate (e.g., can navigate on dusty, concrete surfaces and low lighting) should be documented. Within the "Fleets Navigation and Task" categories, there are several scenarios and the one most relevant to experiments within this paper are that vehicles use or don't use traffic zones and require or don't require permission to enter zones. However, there are cases when the guide requires modifications, for example when two capabilities are combined. In all tests to be performed using the navigation, environmental conditions, and/or the new capabilities standards, the test requestor informs the test administrator of the vehicle configuration, e.g., if safety sensors can be ignored, what the vehicle speed will be, etc. This is important to note as, for example, the vehicle may not be able to perform a task and yet passes the test requestor's criteria.

This paper describes three experiments performed that test the A-UGV Capabilities standard (i.e., does the experiment test whether the A-UGV has or does not have the capability being tested?). Two of the experiments uncovered deficiencies, which led to recommended modifications to the standard. The experiments performed were: 1) two vehicles navigating within the same zone, 2) having transparent obstacles in front of another obstacle, and 3) vehicles using ramps while carrying payloads. These experiments were chosen from a large number of possible scenarios because the researchers hypothesized that they represent typical, complex A-UGV task scenarios that could prove out the validity of the new standard. Experiments and results for the three cases are described with conclusions recommending changes to the A-UGV Capabilities standard. 


\section{Experiments and Results}

Three experiments were conducted at the National Institute of Standards and Technology using one or two autonomous vehicles. The two vehicles were from the same manufacturer but were different models: a cart-transporter and a load carrier [12] ${ }^{1}$. They provided the same obstacle detection, navigation, ramp traversal, mapping, and other configuration capabilities. The first experiment demonstrated that the new A-UGV Capabilities standard properly addresses the many capabilities tested. However, two of the following three experiments (2 and 3) demonstrated that the standard may require improvements. The experiments were performed in an industrial laboratory having a similar environment to warehouses and other manufacturing facilities, although the floor had been clearcoat-sealed making it a lower friction surface than unsealed concrete.

\subsection{Experiment 1: A-UGVs within the same zone}

In a previous experiment [13], the same two A-UGVs used in the present experiments operated in an open area (i.e., not a thin, walled-off corridor as in the present scenario) to test their performance with moving obstacles (i.e., another vehicle). Similar to the prior experiment, the two vehicles were programmed with simultaneous opposing routes. For Experiment 1, the ASTM F3244 Navigation: Defined Area standard [14] was followed, which confined the space in which the two A-UGVs could navigate. The experiment intended to test the A-UGV Capabilities standard in three main areas: navigation, obstacle avoidance, and fleets.

Figure 2 shows the experimental setup. The experimental setup included two different types and sizes of A-UGV: the load carrier measuring $700 \mathrm{~mm} \mathrm{~L} \mathrm{x} 610 \mathrm{~mm} \mathrm{~W}$ x $1395 \mathrm{~mm} \mathrm{H}$ and the cart mover measuring $895 \mathrm{~mm} \mathrm{~L} \mathrm{x} 1075 \mathrm{~mm} \mathrm{~W}$ x $1395 \mathrm{~mm} \mathrm{H}$. Except for their sizes, all other A-UGV configurations needed for the three experiments were identical, e.g., sensors, sensor locations, controller.

The defined area was bounded by straight walls of matte-black painted wooden panels. The distance between the goal points was $8500 \mathrm{~mm}$ and the path width was $3430 \mathrm{~mm}$. The two AUGVs had side clearances of $200 \mathrm{~mm}$, and considering this, a width of $2485 \mathrm{~mm}$ was required to pass without collision. The two A-UGV fronts faced each other with each vehicle goal just behind the other vehicle at their start (as shown in Fig. 2). Each A-UGV started at the same time and was to arrive at the opposite goal by navigating its own self-determined path. The test was repeated a total of 60 times, where each vehicle navigated 30 times in each direction. The success of each A-UGV, the number of e-stops for each A-UGV, and the number of route changes for each A-UGV were recorded. For this experiment, to successfully pass as a capability meant that: 1) no collisions with walls or the other vehicle occurred, 2) the A-UGVs did not become stuck as both A-UGVs had their independent tasks to achieve, and 3) both AUGVs successfully reached their goals.

\footnotetext{
${ }^{1}$ Commercial products are identified in this paper to foster understanding. This does not imply recommendation or endorsement by NIST, nor that the products identified are necessarily the best available for the purpose.
} 


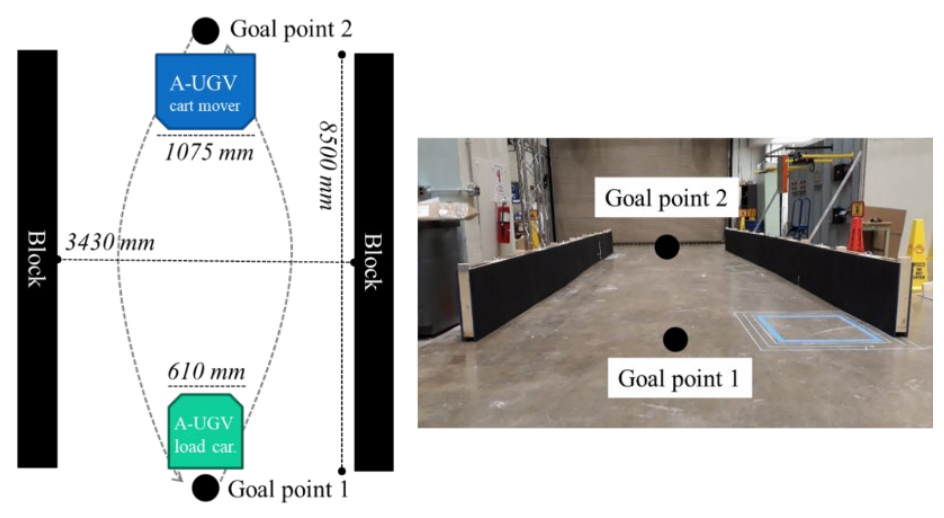

The experimental results for Experiment 1 are shown in Table 1 with notable analysis included as follows. Ideally, each A-UGV made only one path change (i.e., each vehicle detected the other vehicle and re-routed around it) to reach their goal successfully (i.e., completed their task) without stopping. During the experiment, the ideal case occurred 14 times out of the 60 trials. Alternatively, there were 10 trials when the loader changed paths more than the cart mover, and 36 trials when the cart mover changed paths more than the loader.

Table 1. Experiment 1 test results showing the A-UGV success rate and test occurrences.

\begin{tabular}{cccc}
\hline & $\begin{array}{c}\text { \# of } \\
\text { Successes }\end{array}$ & $\begin{array}{c}\text { \# of Path } \\
\text { Changes } \\
\text { during a test }\end{array}$ & $\begin{array}{c}\text { \# of Stops } \\
\text { during a } \\
\text { test }\end{array}$ \\
\hline Loader & 60 & Avg: 1.7 & Avg: 0.4 \\
A-UGV & & Max: 7 & Max: 2 \\
Cart Mover & \multirow{2}{*}{59} & $\begin{array}{c}\text { Avg: } 2.4 \\
\text { Max: } 10\end{array}$ & $\begin{array}{c}\text { Avg: } 0.4 \\
\text { Max: } 3\end{array}$ \\
\hline
\end{tabular}

There were no collisions between the A-UGVs. This implies that each A-UGV can safely avoid static and moving obstacles. However, the cart mover failed one test by hitting the wall, perhaps caused by the combination of a narrowed path and a moving obstacle. The test requestor, as they are called in ASTM F45, requesting this test in a real-world scenario must therefore decide how to interpret the wall collision, i.e., is the collision acceptable performance or not?

The driving pattern was divided into two cases as shown in Fig. 3. First, in the left of Fig. 3 the two A-UGVs redirected to opposite sides and in the right, they moved to the same side. Second, if one of the two A-UGVs stopped and the other A-UGV passed the stopped A-UGV, the stopped A-UGV then started again. This occurred multiple times during a test. Without rules provided by a test requestor to pass in a certain way (e.g., to pass only on the right side), the capability to avoid static and moving obstacles and to navigate with in-situ path planning was demonstrated. 


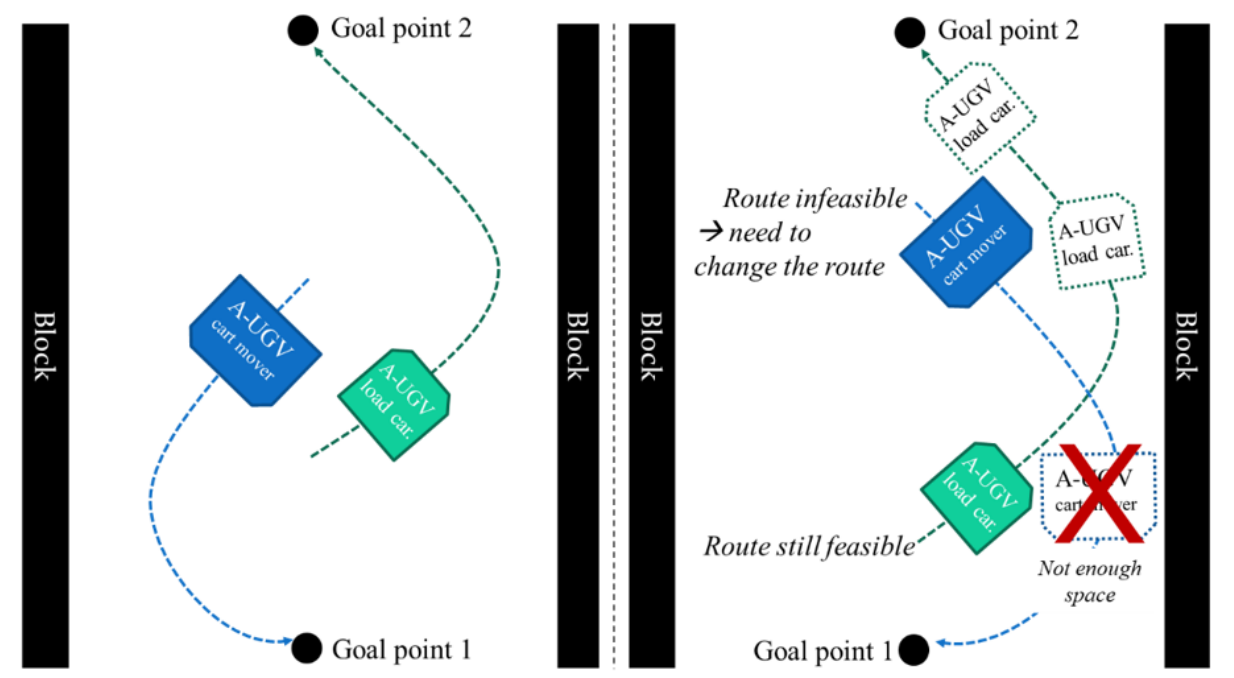

Fig. 3. When two A-UGVs choose different sides (left) and the same side (right).

Analyzing why the cart mover makes more path changes and earlier than the loader may be due to the difference in vehicle width. If the A-UGV detects an obstacle in front of the vehicle, it must decide which side to avoid. The experiment demonstrated that when the two A-UGVs decided to navigate in different directions, there was no problem. When the two A-UGVs navigated in the same direction, the two A-UGVs shifted to one side from the path center. The small A-UGV found a feasible path from the original route, while the large A-UGV needed to generate a new path to the goal as there was not enough space in the original route. Thus, the large A-UGV changed paths more and earlier while the smaller one kept driving.

This case confirmed that two, independently-commanded A-UGVs had the capabilities, among many other capabilities, to successfully perform their navigation tasks while they each: planned their paths in-situ, stopped when the path was obstructed, and navigated around static and moving obstacles. Additionally, if the two, independently-commanded A-UGVs are regarded as a fleet programmed to complete simultaneous tasks within a zone, they also followed the AUGV capabilities standard demonstrating that they can: navigate through the same zone without requiring permissions from or communication with a central controller or the other vehicle. As such, the experiment proved, using the F3244 standard, that the A-UGVs had navigation capabilities and asserted that the A-UGVs had obstacle avoidance and fleet capabilities described in the A-UGV Capabilities.

\subsection{Experiment 2: Transparent obstacles in front of another obstacle}

Transparent curtains are typically installed in warehouses at the opening of a temperaturemaintained room allowing vehicles and people to enter/exit the room. When these curtains are transparent, a person can detect any object, including other people, through the curtain. These curtains may or may not be considered obstacles by humans since they are meant to be penetrated, although they are a divider between two areas. When performing a test, ASTM F3218 should be followed to document the environmental conditions and the test administrator may or may not consider the curtain as an obstacle to the A-UGV, although the curtain should be at least noted on the report. The A-UGV capabilities standard includes a box to check for 
testing the A-UGVs obstacle detection capability. It also requests the user to include a range of obstacles that can be detected. However, it does not include a check box for proving or asserting that an obstacle can be detected through a transparent obstacle.

A-UGVs may or may not be able to detect the curtain or a person or other obstacle through the curtain, yet the vehicle is expected to pass through freely. As such, in a typical scenario, safety or other sensors may be turned off or they may be ignored in order to pass through the curtain. This poses a safety risk should a person or other obstacle be present. Experiment 2 tested the transparent curtain scenario.

Figure 4 (top-left) shows an A-UGV approaching a transparent curtain and Fig. 4 (bottom-left) shows an example with the curtain in front of an obstacle (mock fork tines). The vehicle was commanded to pass through the curtain to a goal several meters beyond the curtain. Experimental results were considered as either a pass (i.e., detected both the curtain and the obstacle) or a fail (i.e., did not detect either the curtain or the obstacle). Additionally, the AUGV should recognize the vicinity, including the curtain as a pass-through area and, with no additional obstacles in the path, should continue through the curtain. Figure 4 (top-right) shows that when the A-UGV approached a transparent curtain, the associated data shows the curtain being detected by the A-UGV sensors. This is the most common issue where the transparent curtain is detected as an obstacle. In this case, the A-UGV does not successfully pass through the transparent curtain and therefore fails. The detect sensor can be temporarily turned off or ignored although this causes a potential safety issue.
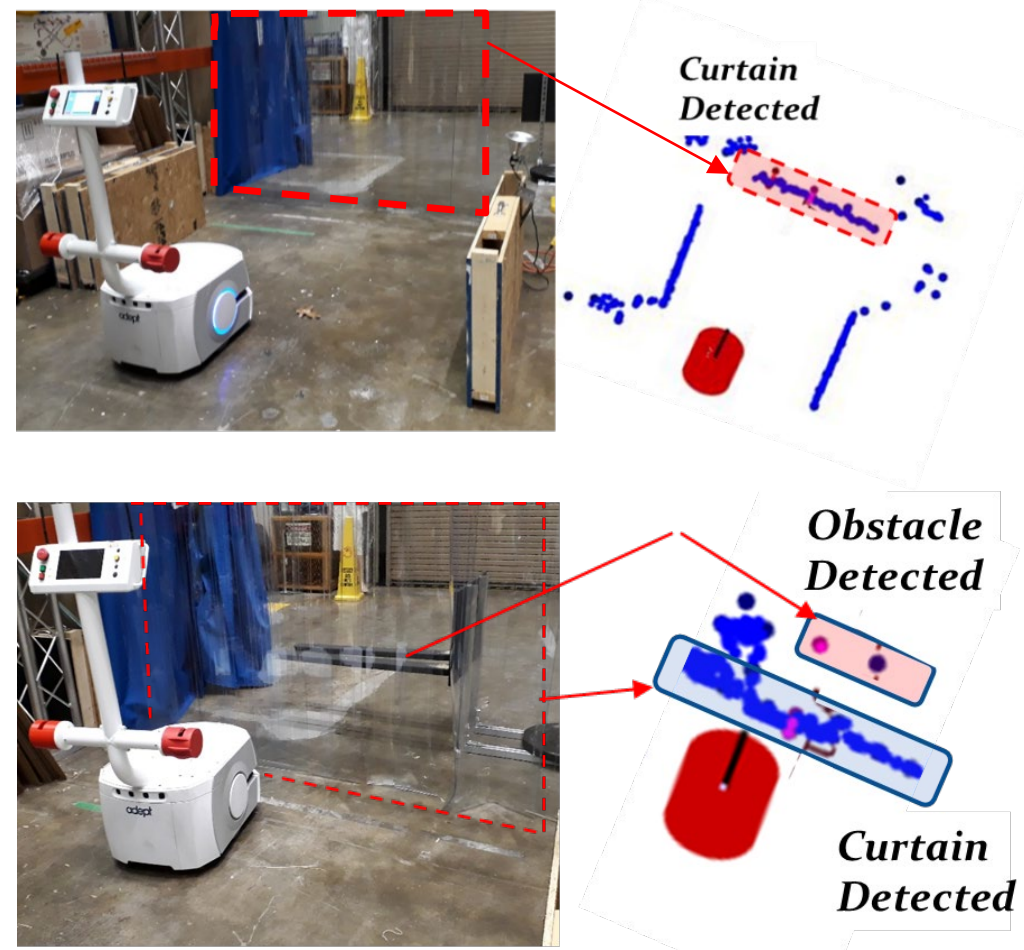

Fig. 4. Transparent curtain (top-left - marked by the red dashed line) is detected (top-right) as an obstacle causing the A-UGV to stop. Obstacle is placed behind a transparent curtain (bottom-left) and both are detected, respectively (Bottom-right). 
Figure 4 (Bottom-right) shows that when an obstacle (mock fork tines) is placed behind the transparent curtain, the A-UGV detected both the curtain and the obstacle. The A-UGV therefore, passed the test although it must, in this case, create a bypass route or communicate to a supervisor (e.g., central controller) that the route is impassable.

Transparent material objects present another issue: the same transparent material could be recognized as an obstacle in some cases. For example, the A-UGV should not go through glass walls and an A-UGV should recognize glass plates stacked as obstacles in the path and avoid them. In other words, the transparent object must be recognized as an obstacle or treated as an environmental factor depending on the situation. As described in Section 1, the test requestor is to request the sensing and other vehicle configurations for the tests to be performed.

When interacting with transparent curtains, A-UGV capabilities can be described as the ability to: detect transparent obstacles, pass through a transparent curtain, detect obstacles through a transparent curtain, and distinguish between obstacles and a transparent curtain. The A-UGV Capabilities standard currently only considers general obstacles like bar, panel, box, sphere, or desk, that are detected by the A-UGV. As such, it is recommended that the A-UGV Capabilities standard should not only describe the variety of obstacles that the vehicle can detect, but also include these obstacle detection combinations.

\subsection{Experiment 3: Loaded A-UGV on a ramp}

Warehouses or manufacturing facilities sometimes task A-UGVs with carrying loads on ramps. The A-UGV Capabilities standard lists the load handling capability, although it does not include in the capability that the load could be carried while the A-UGV is navigating on a ramp. Ramps are described in ASTM F3218 as an environmental condition and in the Capabilities standard, as described in section III, B, bounded conditions in which an A-UGV can perform. However, the bounding conditions do not necessarily mean that the A-UGV can combine capabilities, for example carrying loads in bounded conditions, such as: cold-throughhot temperatures, light-through-high humidity, intense light-through-darkness, and, as in Experiment 3, carrying light-through-heavy loads.

The manufacturer of the A-UGV used in this work specifies full load as $45 \mathrm{~kg}$ on a maximum ramp angle of $5^{\circ}$. However, to fully understand the A-UGV capabilities on ramps, we tested $5^{\circ}$ and $10^{\circ}$ ramps and loads up to $55 \mathrm{~kg}$. Figure 6 shows the experimental setup with $5^{\circ}$ and $10^{\circ}$ ramps to be navigated up to approximately $400 \mathrm{~mm}$ high. The A-UGV type is a load carrier and barbell weights were contained in a box mounted on the vehicle. 


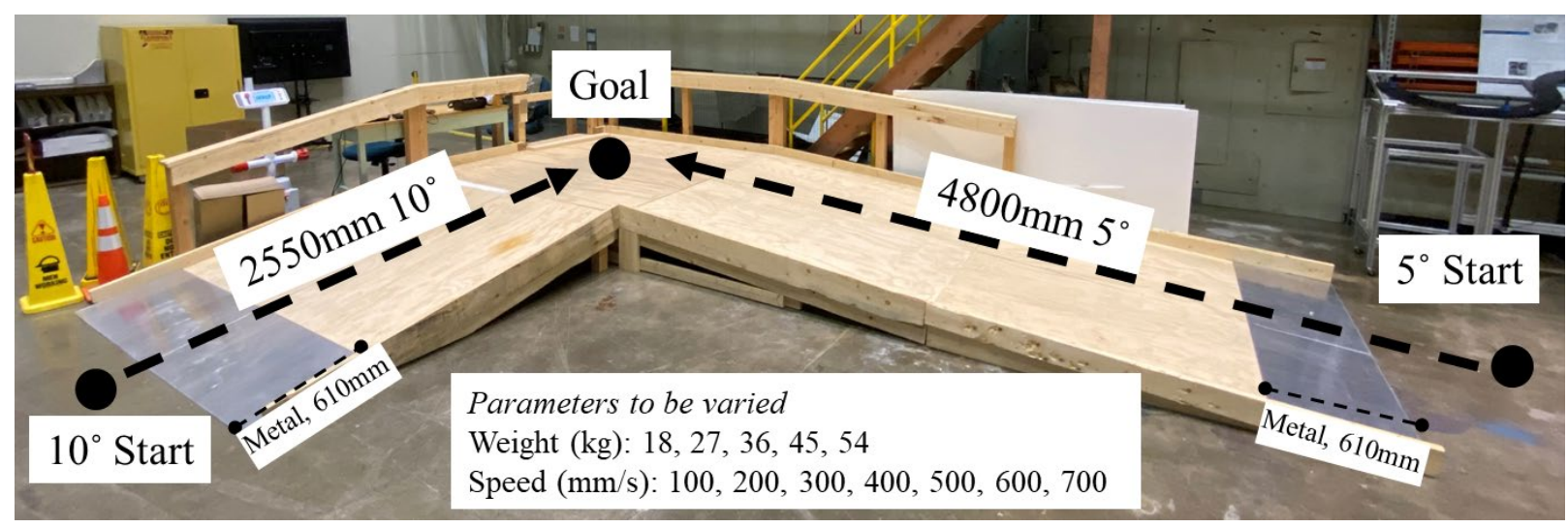

Fig. 5. Summary of experimental setup for a loaded A-UGV while navigating on ramps.

Weights were added in increments of $9 \mathrm{~kg}$ from $18 \mathrm{~kg}$ to $54 \mathrm{~kg}$. The A-UGV speed was also set from $100 \mathrm{~mm} / \mathrm{s}$ to $700 \mathrm{~mm} / \mathrm{s}$ in increments of $100 \mathrm{~mm} / \mathrm{s}$. The A-UGV acceleration was set at $800 \mathrm{~mm} / \mathrm{s}^{2}$. The ramp testbed is made of wood, with $610 \mathrm{~mm}$ of metal floor-to-ramp transitions between the concrete floor and wood ramp to simulate diverse factory flooring. As described at the beginning of this section, the concrete floor was coated with a clear sealer that has a lower coefficient of friction than that of unsealed concrete. Preliminary experiments confirmed that the A-UGV was capable of performing all load and speed combinations on level concrete surfaces.

The experimental procedure was as follows. The A-UGV started $1 \mathrm{~m}$ away from the ramp entrance, transitioned onto the ramp, and navigated up the ramp and to the center of the platform (goal). If the A-UGV arrived at the goal with less than $10 \mathrm{~mm}$ of error, the test was determined to be a success, otherwise it was a failure. The A-UGV drove on each $5^{\circ}$ and $10^{\circ}$ ramp five times for each weight and speed combination and the number of successes was documented.

Again, as described in Section 1, the test requestor specifies the sensing and other vehicle configurations for the tests to be performed. Sensor configurations may be adjusted by the AUGV operator to allow for vehicle use on ramps versus only on level ground surfaces. For example, obstacle detection sensors may be configured to detect but not stop the vehicle from navigating onto ramps where the ramp may be detected as an obstacle. The following configuration was applied to remove the ramp detected as an obstacle and other external factors: 1) the low laser obstacle detection sensor and vertical sensors were ignored (i.e., only the main sensor was used to detect the path and obstacles), 2) the local path fail distance was allowed to be as low as $50 \mathrm{~mm}$.

The experimental results are shown in tables 2 and 3 for the $5^{\circ}$ and $10^{\circ}$ ramps, respectively. Some columns have been aggregated when the results did not change. There were three major driving failure types. First, the A-UGV got stuck as it could not transition onto the ramp when the weight was heavy and the speed was slow. Second, the A-UGV slipped on the metal transition, which also generated many A-UGV position errors. And third, the A-UGV moved 
slower than the commanded navigation speed and the position error gradually increased. For example, the A-UGV controller showed that the vehicle completed its travel before it actually reached the goal. For tests using the $5^{\circ}$ ramp, only the third type of failure occurred.

Table 2. Number of times that the A-UGV reached the goal comparing A-UGV set speed vs. payload on the $5^{\circ}$ ramp.

\begin{tabular}{ccccc}
\hline Speed $\backslash$ Load & $\mathbf{0 , 1 8 , 2 7} \mathbf{~ k g}$ & $\mathbf{3 6} \mathbf{~ k g}$ & $\mathbf{4 5} \mathbf{~ k g}$ & $\mathbf{5 4} \mathbf{~ k g}$ \\
\hline $\mathbf{1 0 0} \mathbf{~ m m} / \mathbf{s}$ & 5 & 0 & 0 & 0 \\
$\mathbf{2 0 0} \mathbf{~ m m} / \mathbf{s}$ & 5 & 5 & 5 & 0 \\
$\mathbf{3 0 0} \mathbf{~ m m} / \mathbf{s}$ & 5 & 5 & 5 & 5 \\
$\mathbf{4 0 0} \mathbf{~ m m} / \mathbf{s}$ & 5 & 5 & 5 & 5 \\
$\mathbf{5 0 0} \mathbf{~ m m} / \mathbf{s}$ & 5 & 5 & 5 & 5 \\
$\mathbf{6 0 0} \mathbf{~ m m} / \mathbf{s}$ & 5 & 5 & 5 & 5 \\
$\mathbf{7 0 0} \mathbf{~ m m} / \mathbf{s}$ & 5 & 5 & 5 & 5 \\
\hline
\end{tabular}

Table 3. Number of times that the A-UGV reached the goal comparing A-UGV set speed vs. payload on the $10^{\circ}$ ramp.

\begin{tabular}{|c|c|c|c|c|}
\hline Speed $\backslash$ Load & $0 \mathrm{~kg}$ & $18 \mathrm{~kg}$ & $27 \mathrm{~kg}$ & $36,45,54 \mathrm{~kg}$ \\
\hline $100 \mathrm{~mm} / \mathrm{s}$ & 5 & 0 & 0 & 0 \\
\hline $200 \mathrm{~mm} / \mathrm{s}$ & 5 & 0 & 0 & 0 \\
\hline $300 \mathrm{~mm} / \mathrm{s}$ & 5 & 2 & 0 & 0 \\
\hline $400 \mathrm{~mm} / \mathrm{s}$ & 5 & 3 & 1 & 0 \\
\hline $500 \mathrm{~mm} / \mathrm{s}$ & 5 & 5 & 2 & 0 \\
\hline $600 \mathrm{~mm} / \mathrm{s}$ & 5 & 5 & 4 & 0 \\
\hline $700 \mathrm{~mm} / \mathrm{s}$ & 5 & 5 & 4 & 0 \\
\hline
\end{tabular}

There were also A-UGV performance changes when it carried weights on ramps. The actual A-UGV speed was limited when the weight was increased. For example, when the A-UGV carried $54 \mathrm{~kg}$, the vehicle would not run at speeds faster than $500 \mathrm{~mm} / \mathrm{s}$ although the speed was commanded to be higher. Therefore, the vehicle can carry heavier loads on level navigation 
surfaces than it can on ramps, which shows that the load carrying capability changes when combined with the bounding environmental (ramp) condition.

As the weight increased, the speed required to climb the ramp also increased. In the case of the $5^{\circ} \mathrm{ramp}$, it was confirmed that the vehicle could traverse the ramp with the $54 \mathrm{~kg}$ weight at a set speed of $300 \mathrm{~mm} / \mathrm{s}$ or faster. In the case of the $10^{\circ} \mathrm{ramp}$, the A-UGV was able to traverse the ramp faster than $500 \mathrm{~mm} / \mathrm{s}$ set speed to carry a maximum load of $18 \mathrm{~kg}$. The A-UGV does not guarantee success if the payload is heavier than $27 \mathrm{~kg}$ carried on the $10^{\circ} \mathrm{ramp}$.

Larger ramp angles can have more effect on A-UGV performance with payload increase. In the case of the $5^{\circ} \mathrm{ramp}$, the A-UGV passed all tests when carrying $18 \mathrm{~kg}$ to $45 \mathrm{~kg}$ at $200 \mathrm{~mm} / \mathrm{s}$ and above. A significant difference was seen only when adding $9 \mathrm{~kg}$ more to the $45 \mathrm{~kg}$ payload which required at least $300 \mathrm{~mm} / \mathrm{s}$ A-UGV speed. On the other hand, there were large differences in the A-UGV speed needed on the $10^{\circ}$ ramp to carry up to $18 \mathrm{~kg}$. And there were also significant differences on the $10^{\circ}$ ramp for the $27 \mathrm{~kg}$ payloads with failures on tests with speeds below $400 \mathrm{~mm} / \mathrm{s}$ and failures for any speed tested with $36 \mathrm{~kg}$ payloads and above.

Through this experiment, it was confirmed that the combination of ramp and weight can be successfully used to evaluate the A-UGV performance. It was also determined that the payload, A-UGV set speed, and ramp angle affect performance, as well as the basic ability to identify and drive on ramps. Heavy payloads affect the A-UGV in many cases, such as when accelerating to the commanded speed, when driving on slippery floor, and when driving upon the floor-to-ramp transition. Based on the experimental results, it is recommended that the AUGV Capabilities standard be modified to include the combination of environmental conditions, such as ramps, with A-UGV payload carrying capabilities.

\section{Conclusions}

A-UGVs are increasingly being used in autonomous tasks across many applications. Their autonomous capabilities must be compared to the task they are required to complete. This study analyzed A-UGV performance for typical facility applications particularly focusing on navigation and obstacle avoidance. This study also considered the facility environment information for manufacturing system design and construction, for example temperature control curtains, ramps, and defined areas. This study tested, through experiments, the application of a new standard guide for documenting some A-UGV Capabilities and provides recommendations for improving the standard. More capabilities can be tested and these tests are recommended for future research.

Testbeds were constructed and, depending on the need, one or two A-UGVs were used to perform three experiments to measure A-UGV performance, including: Experiment 1 - two A-UGVs testing obstacle avoidance when being within the same zone; Experiment 2 - a transparent obstacle in front of another obstacle; and Experiment 3 - an A-UGV with varying payloads on a ramp. The A-UGV Capabilities standard guide allows the user to document each independent capability as proven (with a standard test method), asserted (without a standard test method), untested, or no capability claimed by the vendor. In all three cases, the experiments proved or asserted that the vehicles possessed the capability that was tested, for 
example to navigate through curtains, to navigate on ramps, or to avoid obstacles and act as a fleet.

In Experiment 1, the results showed that the A-UGV Capabilities standard properly addresses cases for the many A-UGV capabilities that were demonstrated during the experiment. However, results from experiments 2 and 3 clearly showed cases in which the bounded environmental conditions require changes based on the tested environment. Therefore, the following recommendations are suggested to enhance the A-UGV Capabilities standard:

1. Combinations of A-UGV capabilities should be included to not only provide bounding environmental conditions, but to also include specific combinations of cases where the vehicle has capabilities. For example, from the experiments performed, the report should also show that the A-UGV tested can detect transparent obstacles, pass through a transparent curtain, detect obstacles through a transparent curtain, and distinguish between obstacles and a transparent curtain.

2. Combinations of A-UGV capabilities should include A-UGV payloads and speeds with varying ramp angles and floor conditions, not just the payload that an A-UGV can carry on level surfaces.

\section{References}

[1] Clark, M.L. There are robots on campus - here's what you need to know. George Mason University, https://www2.gmu.edu/news/2019-01/there-are-robots-campusheres-what-you-need-know, accessed 26 NOV, 2021.

[2] SPARC/RoboHub. Farming with robots. https://robohub.org/farming-with-robots/, accessed 26 NOV, 2021.

[3] Kerr, D. Robots roam hallways of SF's newest hospital, lending a helping hand, https://www.cnet.com/news/robots-give-a-helping-hand-in-san-franciscos-newesthospital/, January 16, 2015, accessed 26 NOV, 2021.

[4] Joosse, M., Evers, V. A (2017) Guide Robot at the Airport: First Impressions. Proc. of the Companion of the 2017 ACM/IEEE Int'l Conf on Human-Robot Interaction, (IEEE Vienna, Austria), pp 149-150. https://doi.org/10.1145/3029798.3038389

[5] Bluebotics, https://bluebotics.com/uvc-disinfection-robot-fights-viruses/, accessed 26 NOV, 2021.

[6] MHI, https://www.mhi.org/mag, accessed 26 NOV, 2021.

[7] Aethon, https://aethon.com/mobile-robots-for-manufacturing/, accessed $26 \mathrm{NOV}$, 2021.

[8] American National Standards Institute, (2019). ANSI/ITSDF B56.5 Safety Standard For Guided Industrial Vehicles, https://www.ansi.org/

[9] ASTM Committee F45 Driverless Automatic Guided Industrial Vehicles, https://www.astm.org/get-involved/technical-committees/committee-f45, accessed 26 NOV, 2021.

[10] Bostelman, R.V. and Messina, E.R. (2019) A-UGV Capabilities-Recommended Guide to Autonomy Levels. 3rd IEEE Int'l Conf. on Robotic Computing, (IEEE, Naples, Italy). https://tsapps.nist.gov/publication/get_pdf.cfm?pub_id=927167 
[11] ASTM F3218-19 Standard Practice for Documenting Environmental Conditions for Utilization with A-UGV Test Methods, https://www.astm.org/get-involved/technicalcommittees/committee-f45, accessed 26 NOV, 2021.

[12] Omron Mobile Robot Specifications, https://assets.omron.com/m/1bf71ca06f961b4b/original/LD-Series-Mobile-RobotDatasheet.pdf, accessed 26 NOV, 2021.

[13] Yoon, S. and Bostelman, R. (2019) Analysis of Automatic through Autonomous Unmanned Ground Vehicles (A-UGVs) Towards Performance Standards. 13th IEEE Int'l Symp. on RObotic and SEnsors Environments (ROSE), (IEEE, Ottawa, ON), pp 1-8. https://doi.org/10.1109/ROSE.2019.8790421

[14] ASTM 3244-17 Standard Test Method for Navigation: Defined Area, https://www.astm.org/get-involved/technical-committees/committee-f45, accessed 26 NOV, 2021. 